\title{
Carathéodory balls in convex complex ellipsoids
}

\author{
by WŁODZIMIERZ ZwONEK (Kraków)
}

\begin{abstract}
We consider the structure of Carathéodory balls in convex complex ellipsoids belonging to few domains for which explicit formulas for complex geodesics are known. We prove that in most cases the only Carathéodory balls which are simultaneously ellipsoids "similar" to the considered ellipsoid (even in some wider sense) are the ones with center at 0 . Nevertheless, we get a surprising result that there are ellipsoids having Carathéodory balls with center not at 0 which are also ellipsoids.
\end{abstract}

1. Introduction. For any domain $D \subset \mathbb{C}^{n}$ and $w, z \in D$ we define

$c_{D}(w, z)=\sup \{\varrho(\varphi(w), \varphi(z)): \varphi: D \rightarrow E$ is a holomorphic mapping $\}$,

where $E$ is the unit disk in $\mathbb{C}$ and $\varrho$ is the Poincaré distance (also called the hyperbolic distance) on $E . c_{D}$ is called the Carathéodory pseudodistance of $D$. We also define

$$
c_{D}^{*}:=\tanh c_{D} .
$$

If $D$ is a bounded domain, then $c_{D}$ is a distance on $D$.

A holomorphic mapping $\varphi: E \rightarrow D$ is called a c-geodesic in $D$ if $c_{D}\left(\varphi\left(\lambda_{1}\right), \varphi\left(\lambda_{2}\right)\right)=\varrho\left(\lambda_{1}, \lambda_{2}\right)$ for any $\lambda_{1}, \lambda_{2} \in E$.

It is well known (see [L]) that if $D$ is a convex, bounded domain, then for any pair of points $(w, z) \in D \times D$ with $w \neq z$ there is a $c$-geodesic $\varphi: E \rightarrow D$ such that $\varphi(0)=w$ and $\varphi\left(c_{D}^{*}(w, z)\right)=z$.

If $D$ is a bounded domain, then for $w \in D$ and $0<r<1$ we define the Carathéodory ball as

$$
B_{c_{D}^{*}}(w, r):=\left\{z \in D: c_{D}^{*}(w, z)<r\right\} .
$$

Below we shall consider the domains

$$
\mathcal{E}(p):=\left\{\left|z_{1}\right|^{2 p_{1}}+\ldots+\left|z_{n}\right|^{2 p_{n}}<1\right\},
$$

1991 Mathematics Subject Classification: Primary 32H15.

Key words and phrases: Carathéodory ball, $c$-geodesic, convex complex ellipsoid.

This paper has been supported by KBN grant No 2 PO3A 06008 . 
where $p=\left(p_{1}, \ldots, p_{n}\right), p_{j}>0$. They are called complex ellipsoids. If $n \geq 2$, then a complex ellipsoid is convex iff $p_{j} \geq 1 / 2$ for $j=1, \ldots, n$. Below we only consider convex ellipsoids.

For $\widetilde{r}, \widetilde{r}>0$ and $w \in \mathbb{C}^{n}$ we also define the $N_{p^{-}}$ellipsoids

$$
\mathcal{E}_{p}(w, \widetilde{r}, \widetilde{r}):=\left\{z \in \mathbb{C}^{n}: N_{p}(z-w, \widetilde{r})<\widetilde{\widetilde{r}}\right\},
$$

where $N_{p}(z, \widetilde{r}):=\left|z_{1} / \widetilde{r}\right|^{2 p_{1}}+\ldots+\left|z_{n} / \widetilde{r}\right|^{2 p_{n}}$.

Note that

$$
\mathcal{E}(p)=\mathcal{E}_{p}(0,1,1)
$$

In case $p_{1}=\ldots=p_{n}$ we have $\mathcal{E}_{p}(w, \widetilde{r}, \widetilde{r})=\mathcal{E}_{p}\left(w, \widetilde{r}(\widetilde{r})^{1 /\left(2 p_{1}\right)}, 1\right)$, therefore in this case without loss of generality considering $N_{p}$-ellipsoids we may restrict ourselves to the ones with $\widetilde{r}=1$. Moreover, these ellipsoids are balls in the sense of the norm $\|z\|_{2 p_{1}}=\left(\left|z_{1}\right|^{2 p_{1}}+\ldots+\left|z_{n}\right|^{2 p_{1}}\right)^{1 /\left(2 p_{1}\right)}$ (certainly if $\left.p_{1} \geq 1 / 2\right)$.

In the general case the condition $N_{p}(z, \widetilde{r})=1$ means that the Minkowski function of $\mathcal{E}(p)$ equals $\widetilde{r}$ at $z$. Therefore, $\mathcal{E}_{p}(w, \widetilde{r}, 1)$ is the "Minkowski ball" with center at $w$ and radius $\widetilde{r}$.

The aim of our paper is the following theorem.

Theorem 1. Let $p=\left(p_{1}, \ldots, p_{n}\right)$, where $n \geq 2$ and $p_{j} \geq 1 / 2$ for $j=$ $1, \ldots, n$. Let $w \in \mathcal{E}(p)$ and $0<r<1$. Assume that

$$
B_{c_{\mathcal{E}(p)}^{*}}(w, r)=\mathcal{E}_{p}(\widetilde{w}, \widetilde{r}, \widetilde{r})
$$

for some $\widetilde{w} \in \mathcal{E}(p), \widetilde{r}, \widetilde{r}>0$. Then

- if $\left(p_{j} \neq 1, j=1, \ldots, n\right)$ or $p_{1}=\ldots=p_{n}=1$ then $w=0$.

In the general case the above implication does not hold. We have

- if $n=2, p_{2}=1$ and $p_{1}=1 / 2$ then any ball $B_{\mathcal{E}_{\mathcal{E}(p)}^{*}}\left(\left(0, w_{2}\right), r\right)$ is an $N_{p}$-ellipsoid with $\widetilde{\widetilde{r}}=1$,

although

- if $n=2, p_{2}=1, p_{1}>1 / 2, \widetilde{\widetilde{r}}=1$ and (1) holds then $w=0$.

Moreover, if $1 \neq p_{1} \geq 1 / 2, p_{2}=1$ and $n=2$ then any ball $B_{c_{\mathcal{E}(p)}^{*}}\left(\left(0, w_{2}\right), r\right)$ is an $N_{p}$-ellipsoid with some $\widetilde{\widetilde{r}}>0$.

The above theorem is a generalization of earlier results. In the case of the unit ball (i.e. $p_{1}=\ldots=p_{n}=1$ ) the theorem has been proven in [R]. For $n=2$ and $p_{1}=p_{2}=1 / 2$ the theorem has been proven in [Sc]; a generalization of Schwarz's result $\left(n \geq 2\right.$ arbitrary, $\left.p_{1}=\ldots=p_{n}=1 / 2\right)$ can be found in $[\mathrm{Sr}]$ and $[\mathrm{Z}]$. The more general case $2 p_{1}=\ldots=2 p_{n}=: q>2$, 
where $q$ is not an even integer, has been obtained in [Sc-Sr]. Our theorem gives in particular a direct generalization of the last result.

Recall that the Carathéodory balls in $\mathcal{E}(p)$ with center at 0 are sets of the type $\mathcal{E}_{p}(0, r, 1)$, so the natural generalization of the theorems known so far (see $[\mathrm{Sc}],[\mathrm{Sr}],[\mathrm{Z}],[\mathrm{Sc}-\mathrm{Sr}]$ ) would be the following statement: The only Carathéodory balls in $\mathcal{E}(p)$ which are simultaneously ellipsoids of the type $\mathcal{E}_{p}(\widetilde{w}, \widetilde{r}, 1)$, are the ones with center at 0 . As we see in Theorem 1 this statement is true if $p_{1}=\ldots=p_{n}$ but fails to hold in case $p_{1}=1 / 2, p_{2}=1$. This justifies considering the ellipsoids $\mathcal{E}_{p}(\widetilde{w}, \widetilde{r}, \widetilde{r})$ not necessarily with $\widetilde{\widetilde{r}}=1$.

The convex complex ellipsoids are among few domains for which explicit formulas for complex geodesics are known. From Theorem 1 we obtain a better understanding of the geometry of ellipsoids with respect to the Carathéodory distance; we see that it is quite different from the "natural" geometry of the ellipsoids.

Observe that Theorem 1 is no longer true if $n=1$ (i.e. it is not true in the unit disk $E$ ). Moreover, we have the following characterization of the Carathéodory disks in $E$ (see e.g. [Sc]):

$$
\varrho(z, a)=\tanh ^{-1} r \quad \text { iff } \quad|z-b|=R, \quad \text { for } a, b, z \in E,
$$

where

$$
b=a \frac{1-r^{2}}{1-r^{2}|a|^{2}} \quad \text { and } \quad R=r \frac{1-|a|^{2}}{1-r^{2}|a|^{2}} .
$$

We prove Theorem 1 combining the methods from $[\mathrm{Sc}],[\mathrm{Sr}],[\mathrm{Z}]$ and [Sc-Sr] and also making use of the following characterization of $c$-geodesics in convex complex ellipsoids.

TheOREM 2 (see [JPZ] and [JP]). Let $\mathcal{E}(p)$ be a convex complex ellipsoid. $A$ bounded holomorphic mapping $\varphi=\left(\varphi_{1}, \ldots, \varphi_{n}\right): E \rightarrow \mathbb{C}^{n}$ is a c-geodesic in $\mathcal{E}(p)$ if and only if either

$$
\varphi_{j}(\lambda)=a_{j}\left(\frac{\lambda-\alpha_{j}}{1-\bar{\alpha}_{j} \lambda}\right)^{r_{j}}\left(\frac{1-\bar{\alpha}_{j} \lambda}{1-\bar{\alpha}_{0} \lambda}\right)^{1 / p_{j}}
$$

or

$$
\varphi_{j}(\lambda)=0,
$$

where $r_{j} \in\{0,1\}$ and $a_{j} \in \mathbb{C}_{*}$ for $j=1, \ldots, n, \alpha_{0} \in E, \alpha_{j} \in E$ for $j$ such that $r_{j}=1, \alpha_{j} \in \bar{E}$ for $j$ such that $r_{j}=0$, and the following relations hold (in the case (3) we put $\alpha_{j}:=0, a_{j}:=0, r_{j}:=0$ ):

$$
\alpha_{0}=\sum_{j=1}^{n}\left|a_{j}\right|^{2 p_{j}} \alpha_{j}
$$




$$
1+\left|\alpha_{0}\right|^{2}=\sum_{j=1}^{n}\left|a_{j}\right|^{2 p_{j}}\left(1+\left|\alpha_{j}\right|^{2}\right) .
$$

The case where for any $j=1, \ldots, n$ the mapping $\varphi_{j}$ is either of the form (3) or of the form (2) with $r_{j}=0$ and $\alpha_{j}=\alpha_{0}$ is excluded. The branches of powers are taken so that $1^{1 / p_{j}}=1$. Moreover, for any pair of different points we have the uniqueness of complex geodesics passing through these points, up to automorphisms of $E$.

2. Auxiliary results. Below we present a theorem, which is a simple consequence of Theorem 2 .

Theorem 3. Let $z:=\left(z_{1}, \ldots, z_{k}, z_{k+1}, \ldots, z_{n}\right)$ and $w:=\left(w_{1}, \ldots, w_{k}\right.$, $\left.z_{k+1}, \ldots, z_{n}\right)$ be distinct points in a convex ellipsoid $\mathcal{E}(p), n \geq 2$. Put $\gamma:=$ $1-\sum_{j=k+1}^{n}\left|z_{j}\right|^{2 p_{j}}$. Let $\varphi$ be a c-geodesic in $\mathcal{E}\left(p_{1}, \ldots, p_{k}\right)$ joining $\left(w_{1} / \gamma^{1 /\left(2 p_{1}\right)}, \ldots, w_{k} / \gamma^{1 /\left(2 p_{k}\right)}\right)$ to $\left(z_{1} / \gamma^{1 /\left(2 p_{1}\right)}, \ldots, z_{k} / \gamma^{1 /\left(2 p_{k}\right)}\right)$. Then

$$
\widetilde{\varphi}(\lambda):=\left(\gamma^{1 /\left(2 p_{1}\right)} \varphi_{1}, \ldots, \gamma^{1 /\left(2 p_{k}\right)} \varphi_{k}, z_{k+1}, \ldots, z_{n}\right)
$$

is a c-geodesic joining $w$ to $z$. Consequently,

$$
\begin{aligned}
& c_{\mathcal{E}(p)}(w, z) \\
& \quad=c_{\mathcal{E}\left(p_{1}, \ldots, p_{k}\right)}\left(\left(\frac{w_{1}}{\gamma^{1 /\left(2 p_{1}\right)}}, \ldots, \frac{w_{k}}{\gamma^{1 /\left(2 p_{k}\right)}}\right),\left(\frac{z_{1}}{\gamma^{1 /\left(2 p_{1}\right)}}, \ldots, \frac{z_{k}}{\gamma^{1 /\left(2 p_{k}\right)}}\right)\right) .
\end{aligned}
$$

Pro of. Without loss of generality we may assume that $z_{k+1}, \ldots, z_{n} \neq 0$ and $\left|z_{j}\right|+\left|w_{j}\right|>0$ for $j=1, \ldots, k$. Let the numbers $a_{1}, \ldots, a_{k}, \alpha_{0}, \alpha_{1}, \ldots$ $\ldots, \alpha_{k}, r_{1}, \ldots, r_{k}$ correspond to $\varphi$ as in Theorem 2. Then (see (4) and (5))

$$
\begin{gathered}
\alpha_{0}=\left|a_{1}\right|^{2 p_{1}} \alpha_{1}+\ldots+\left|a_{k}\right|^{2 p_{k}} \alpha_{k}, \\
1+\left|\alpha_{0}\right|^{2}=\left|a_{1}\right|^{2 p_{1}}\left(1+\left|\alpha_{1}\right|^{2}\right)+\ldots+\left|a_{k}\right|^{2 p_{k}}\left(1+\left|\alpha_{k}\right|^{2}\right) .
\end{gathered}
$$

The mapping $\widetilde{\varphi}$ is described by the coefficients $\widetilde{a}_{1}, \ldots, \widetilde{a}_{n}, \widetilde{\alpha}_{0}, \widetilde{\alpha}_{1}, \ldots, \widetilde{\alpha}_{n}$ and $\widetilde{r}_{1}, \ldots, \widetilde{r}_{n}$ (as in Theorem 2), where

$$
\begin{aligned}
& \widetilde{a}_{j}:= \begin{cases}\gamma^{1 /\left(2 p_{j}\right)} a_{j} & \text { for } j=1, \ldots, k, \\
z_{j} & \text { for } j=k+1, \ldots, n,\end{cases} \\
& \widetilde{\alpha}_{j}:= \begin{cases}\alpha_{j} & \text { for } j=1, \ldots, k, \\
\alpha_{0} & \text { if } j=0 \text { or } j=k+1, \ldots, n,\end{cases} \\
& \widetilde{r}_{j}:= \begin{cases}r_{j} & \text { for } j=1, \ldots, k, \\
0 & \text { for } j=k+1, \ldots, n,\end{cases}
\end{aligned}
$$

To prove that $\widetilde{\varphi}$ is a $c$-geodesic we verify the properties (4) and (5). 
Note that in view of (6),

$$
\begin{aligned}
\sum_{j=1}^{n}\left|\widetilde{a}_{j}\right|^{2 p_{j}} \widetilde{\alpha}_{j} & =\gamma\left(\left|a_{1}\right|^{2 p_{1}} \alpha_{1}+\ldots+\left|a_{k}\right|^{2 p_{k}} \alpha_{k}\right)+\sum_{j=k+1}^{n}\left|z_{j}\right|^{2 p_{j}} \alpha_{0} \\
& =\gamma \alpha_{0}+\alpha_{0} \sum_{j=k+1}^{n}\left|z_{j}\right|^{2 p_{j}}=\alpha_{0}=\widetilde{\alpha}_{0},
\end{aligned}
$$

and in view of (7),

$$
\begin{aligned}
\sum_{j=1}^{n}\left|\widetilde{a}_{j}\right|^{2 p_{j}}\left(1+\left|\widetilde{\alpha}_{j}\right|^{2}\right)= & \gamma\left(\left|a_{1}\right|^{2 p_{1}}\left(1+\left|\alpha_{1}\right|^{2}\right)+\ldots+\left|a_{k}\right|^{2 p_{k}}\left(1+\left|\alpha_{k}\right|^{2}\right)\right) \\
& +\sum_{j=k+1}^{n}\left|z_{j}\right|^{2 p_{j}}\left(1+\left|\alpha_{0}\right|^{2}\right) \\
= & \gamma\left(1+\left|\alpha_{0}\right|^{2}\right)+\left(1+\left|\alpha_{0}\right|^{2}\right) \sum_{j=k+1}^{n}\left|z_{j}\right|^{2 p_{j}}=1+\left|\widetilde{\alpha}_{0}\right|^{2} .
\end{aligned}
$$

This completes the proof of the theorem.

As an immediate consequence of Theorem 2 we also get the following lemma.

Lemma 4. Let $a \in \mathcal{E}(p)$ and $\lambda a \in \mathcal{E}(p)$, where $\lambda \in \mathbb{C}$ and $\mathcal{E}(p)$ is a convex ellipsoid. Then

$$
c_{\mathcal{E}(p)}(\lambda a, a)=\varrho(h(a), \lambda h(a)),
$$

where $h$ is the Minkowski function of $\mathcal{E}(p)$.

Below we formulate and prove some generalization, in case $n=2$, of a result from $[\mathrm{Sc}-\mathrm{Sr}]$.

Proposition 5. Let $\lambda_{1}, \lambda_{2}>0, r_{1}, r_{2}>0, \theta_{1}, \theta_{2} \in \mathbb{R}$. Let $p=\left(p_{1}, p_{2}\right)$, where $p_{1}, p_{2}>0$ and $p_{1}$ and $p_{2}$ are not both equal to 1 . Assume that for $t \in \mathbb{R}$

$$
\lambda_{1}\left|r_{1} e^{i\left(t+\theta_{1}\right)}-a_{1}\right|^{2 p_{1}}+\lambda_{2}\left|r_{2} e^{i\left(t+\theta_{2}\right)}-a_{2}\right|^{2 p_{2}} \equiv \text { const } .
$$

Then $a_{1}=a_{2}=0$.

Proof. Suppose that $\left(a_{1}, a_{2}\right) \neq 0$. The only problem is with the case $a_{1}, a_{2} \neq 0$. Without loss of generality we may assume that $r_{1}=r_{2}=1$, $a_{1}, a_{2}>0$ and $\theta_{1}=0$. Therefore we have, for $t \in \mathbb{R}$,

$$
\lambda_{1}\left|e^{i t}-a_{1}\right|^{2 p_{1}}+\lambda_{2}\left|e^{i(t+\theta)}-a_{2}\right|^{2 p_{2}} \equiv \text { const },
$$

where $\theta \in[0,2 \pi)$

Putting $b_{j}:=\frac{1+a_{j}^{2}}{2 a_{j}}, \lambda_{j}^{\prime}:=\lambda_{j}\left(2 a_{j}\right)^{p_{j}}, j=1,2$, we have

$$
\lambda_{1}^{\prime}\left(b_{1}-\cos (t)\right)^{p_{1}}+\lambda_{2}^{\prime}\left(b_{2}-\cos (t+\theta)\right)^{p_{2}} \equiv \text { const },
$$


where additionally

$$
b_{j} \geq 1 \quad \text { for } j=1,2 .
$$

First we prove that both summands in (8) must be real analytic in $\mathbb{R}$. Suppose that the first one is not. That is only possible when $p_{1} \notin \mathbb{N}$ and $b_{1}=1$ (see (9)). Then it is not real analytic at 0 . But this means that the second summand cannot be real analytic for $t=0$, so $\theta=0$ and $b_{2}=1$ (see $(9)$ ). But this leads us to the following equality for $t \in \mathbb{R}$ :

$$
\lambda_{1}^{\prime}(1-\cos (t))^{p_{1}}+\lambda_{2}^{\prime}(1-\cos (t))^{p_{2}} \equiv \mathrm{const},
$$

which cannot hold - a contradiction.

Therefore

$$
\left(p_{j} \in \mathbb{N} \text { or } b_{j}>1\right) \quad \text { for } j=1,2 .
$$

Let us differentiate (8) with respect to $t$ (in view of the above considerations we have below no expression of type $\infty \cdot 0)$ :

(10) $\lambda_{1}^{\prime} p_{1}\left(b_{1}-\cos (t)\right)^{p_{1}-1} \sin (t)+\lambda_{2}^{\prime} p_{2}\left(b_{2}-\cos (t+\theta)\right)^{p_{2}-1} \sin (t+\theta) \equiv 0$.

Put $t=0$. Then from (10) we get $\left(b_{2}-\cos (\theta)\right)^{p_{2}-1} \sin (\theta)=0$. Consequently,

$$
\left(b_{2}=1, \theta=0\right) \text { or } \theta=0 \text { or } \theta=\pi .
$$

If $\theta=0$ then from (10) we get

$$
\lambda_{1}^{\prime} p_{1}\left(b_{1}-\cos (t)\right)^{p_{1}-1}+\lambda_{2}^{\prime} p_{2}\left(b_{2}-\cos (t)\right)^{p_{2}-1} \equiv 0
$$

for $t \in \mathbb{R}$ - a contradiction.

If $\theta=\pi$ then from (10) we get

$$
\lambda_{1}^{\prime} p_{1}\left(b_{1}-\cos (t)\right)^{p_{1}-1}=\lambda_{2}^{\prime} p_{2}\left(b_{2}+\cos (t)\right)^{p_{2}-1}
$$

for $t \in \mathbb{R}$, so

$$
\frac{\lambda_{1}^{\prime} p_{1}\left(b_{1}-\cos (t)\right)^{p_{1}-1}}{\lambda_{2}^{\prime} p_{2}\left(b_{2}+\cos (t)\right)^{p_{2}-1}} \equiv \text { const },
$$

which, as one can easily prove (e.g. differentiating with respect to $t$ ), holds only if $p_{1}=p_{2}=1-$ a contradiction.

To prove Theorem 1 we shall need one more lemma.

Lemma 6. Let $w, \widetilde{w} \in \mathcal{E}(p), \widetilde{r}, \widetilde{r}>0,1>r>0, n \geq 2$ and $p_{j} \geq 1 / 2$, $j=1, \ldots, n$. Assume that

$$
\partial B_{c_{\mathcal{E}(p)}^{*}}(w, r) \subset \partial \mathcal{E}_{p}(\widetilde{w}, \widetilde{r}, \widetilde{\widetilde{r}}) .
$$

Then

$$
\mathcal{E}_{p}(\widetilde{w}, \widetilde{r}, \widetilde{r})=B_{c_{\mathcal{E}(p)}^{*}}(w, r) .
$$

Proof. First we prove that

$$
w \in \mathcal{E}_{p}(\widetilde{w}, \widetilde{r}, \widetilde{r}), \quad \widetilde{w} \in B_{c_{\mathcal{E}(p)}^{*}}(w, r) .
$$


Suppose that $w \notin \mathcal{E}_{p}(\widetilde{w}, \widetilde{r}, \widetilde{\widetilde{r}})$. Then $N_{p}(w-\widetilde{w}, \widetilde{r}) \geq \widetilde{\widetilde{r}}$ and consequently there is $t \in(0,1]$ such that $\widetilde{w}-t(\widetilde{w}-w)=t w+(1-t) \widetilde{w} \in \partial \mathcal{E}_{p}(\widetilde{w}, \widetilde{r}, \widetilde{r})$. The continuity of the Carathéodory distance gives us also the existence of some $s<0$ such that $\widetilde{w}+(s-1)(\widetilde{w}-w)=w+s(\widetilde{w}-w) \in \partial B_{c_{\mathcal{E}(p)}^{*}}(w, r) \subset$ $\partial \mathcal{E}_{p}(\widetilde{w}, \widetilde{r}, \widetilde{r})$. So there are two distinct points lying on $\widetilde{w}+\mathbb{R}_{+}(w-\widetilde{w})$, which belong to $\partial \mathcal{E}_{p}(\widetilde{w}, \widetilde{r}, \widetilde{r})$ - a contradiction.

Suppose that $\widetilde{w} \notin B_{C_{\mathcal{E}(p)}^{*}}(w, r)$. Then continuity of the Carathéodory distance yields the existence of $t \in[0,1)$ such that $\widetilde{w}-t(\widetilde{w}-w)=t w+$ $(1-t) \widetilde{w} \in \partial B_{c_{\mathcal{E}(p)}^{*}}(w, r) \subset \partial \mathcal{E}_{p}(\widetilde{w}, \widetilde{r}, \widetilde{r})$. As before we get the existence of a second point from $\partial \mathcal{E}_{p}(\widetilde{w}, \widetilde{r}, \widetilde{r})$ lying on $\widetilde{w}+\mathbb{R}_{+}(w-\widetilde{w})$ - a contradiction.

We now prove the inclusion

$$
B_{\mathcal{E}_{\mathcal{E}(p)}^{*}}(w, r) \subset \mathcal{E}_{p}(\widetilde{w}, \widetilde{r}, \widetilde{\widetilde{r}}) .
$$

Take $z \in B_{c_{\mathcal{E}(p)}^{*}}(w, r)$. Then there are $0 \leq s<r$ and a complex geodesic $\varphi: E \rightarrow \mathcal{E}(p)$ such that $\varphi(0)=w$ and $\varphi(s)=z$. Define

$$
h: E \ni \lambda \rightarrow N_{p}(\varphi(\lambda)-\widetilde{w}, \widetilde{r}) \in \mathbb{R} .
$$

Then $h$ is a subharmonic function. Since $\varphi(0)=w \in \mathcal{E}_{p}(\widetilde{w}, \widetilde{r}, \widetilde{r})$, we get $h(0)<\widetilde{\widetilde{r}}$.

For $\lambda$ with $|\lambda|=r$ we have $c_{\mathcal{E}(p)}^{*}(\varphi(\lambda), w)=r$, so $\varphi(\lambda) \in \partial B_{c_{\mathcal{E}(p)}^{*}}(w, r) \subset$ $\partial \mathcal{E}_{p}(\widetilde{w}, \widetilde{r}, \widetilde{r})$. Consequently, $h(\lambda)=\widetilde{\widetilde{r}}$ for $|\lambda|=r$. But the maximum principle for subharmonic functions implies that

$$
h(\lambda)<\widetilde{\widetilde{r}} \text { for }|\lambda|<r
$$

(remember that $h(0)<\widetilde{r}$ ). This completes the proof of the first inclusion.

To get the inverse inclusion, suppose that there is $z \in \mathcal{E}_{p}(\widetilde{w}, \widetilde{r}, \widetilde{r}) \backslash$ $B_{c_{\mathcal{E}(p)}^{*}}(w, r)$. Since $\widetilde{w} \in B_{c_{\mathcal{E}(p)}^{*}}(w, r)$ and the function $c_{\mathcal{E}(p)}^{*}$ is continuous we get the existence of $t \in[0,1)$ such that

$$
z^{\prime}:=t \widetilde{w}+(1-t) z \in \partial B_{c_{\mathcal{E}(p)}^{*}}(w, r) \subset \partial \mathcal{E}_{p}(\widetilde{w}, \widetilde{r}, \widetilde{r})
$$

but from the definition of $N_{p}$ we get

$$
N_{p}\left(z^{\prime}-\widetilde{w}, \widetilde{r}\right) \leq N_{p}(z-\widetilde{w}, \widetilde{r})<\widetilde{r}
$$

-a contradiction.

3. Proof of Theorem 1. As already mentioned, the theorem is known in case $p_{1}=\ldots=p_{n}=1$ (see $[\mathrm{R}]$ ), so in the sequel we exclude this case.

First we prove the theorem for $n=2$. Assume now that $p_{1} \neq 1$ or $p_{2} \neq 1$. Assume that (1) holds for some $w=\left(w_{1}, w_{2}\right)$. As the case $w=0$ is trivial, 
we assume that $w \neq 0$ and there are $\widetilde{w}, r, \widetilde{r}, \widetilde{r}$ such that

$$
\partial B_{c_{\mathcal{E}(p)}^{*}}(w, r)=\partial \mathcal{E}_{p}(\widetilde{w}, \widetilde{r}, \widetilde{r}) .
$$

We consider some one-dimensional subsets of $\partial B_{c_{\mathcal{E}(p)}^{*}}(w, r)$, which correspond to the following subsets of $\mathbb{C}$ :

$$
\begin{aligned}
A_{1} & :=\left\{\zeta \in \mathbb{C}:\left(\zeta, w_{2}\right) \in \partial B_{c_{\mathcal{E}(p)}^{*}}(w, r)\right\}, \\
A_{2} & :=\left\{\zeta \in \mathbb{C}:\left(w_{1}, \zeta\right) \in \partial B_{c_{\mathcal{E}(p)}^{*}}(w, r)\right\}, \\
B & :=\left\{\zeta \in \mathbb{C}: \zeta w \in \partial B_{c_{\mathcal{E}(p)}^{*}}(w, r)\right\} .
\end{aligned}
$$

Observe that for $\zeta \in A_{1}$ we have, in view of Theorem 3 ,

$\tanh ^{-1} r=c_{\mathcal{E}(p)}\left(\left(\zeta, w_{2}\right), w\right)=\varrho\left(\frac{\zeta}{\left(1-\left|w_{2}\right|^{2 p_{2}}\right)^{1 /\left(2 p_{1}\right)}}, \frac{w_{1}}{\left(1-\left|w_{2}\right|^{2 p_{2}}\right)^{1 /\left(2 p_{1}\right)}}\right)$,

which implies that $\zeta /\left(1-\left|w_{2}\right|^{2 p_{2}}\right)^{1 /\left(2 p_{1}\right)}$ lies on the hyperbolic circle with center at $w_{1} /\left(1-\left|w_{2}\right|^{2 p_{2}}\right)^{1 /\left(2 p_{1}\right)}$ and radius $\tanh ^{-1} r$. But this means, in view of the description of the Carathéodory disks in $E$, that (we can analogously proceed with $A_{2}$ )

$$
\begin{aligned}
& A_{j}=\left\{\zeta: \zeta=\frac{\left(1-r^{2}\right)\left(1-\left|w_{3-j}\right|^{2 p_{3-j}}\right)^{1 / p_{j}}}{\left(1-\left|w_{3-j}\right|^{2 p_{j}}\right)^{1 / p_{j}}-r^{2}\left|w_{j}\right|^{2}} w_{j}\right. \\
& \left.\quad+r\left(1-\left|w_{3-j}\right|^{2 p_{3-j}}\right)^{1 /\left(2 p_{j}\right)} \frac{\left(1-\left|w_{3-j}\right|^{2 p_{3-j}}\right)^{1 / p_{j}}-\left|w_{j}\right|^{2}}{\left(1-\left|w_{3-j}\right|^{2 p_{3-j}}\right)^{1 / p_{j}}-r^{2}\left|w_{j}\right|^{2}} e^{i \theta}, 0 \leq \theta \leq 2 \pi\right\}
\end{aligned}
$$

for $j=1,2$.

But from the equality (11) we know that for $\zeta \in A_{j}, j=1,2$,

$$
\widetilde{\widetilde{r}}=\left|\frac{\zeta-\widetilde{w}_{j}}{\widetilde{r}}\right|^{2 p_{j}}+\left|\frac{w_{3-j}-\widetilde{w}_{3-j}}{\widetilde{r}}\right|^{2 p_{3-j}} .
$$

From the form of $A_{j}$ we get

$$
\widetilde{w}_{j}=\frac{\left(1-r^{2}\right)\left(1-\left|w_{3-j}\right|^{2 p_{3-j}}\right)^{1 / p_{j}}}{\left(1-\left|w_{3-j}\right|^{2 p_{3-j}}\right)^{1 / p_{j}}-r^{2}\left|w_{j}\right|^{2}} w_{j}, \quad j=1,2 .
$$

In particular, $w_{j}=0$ if and only if $\widetilde{w}_{j}=0$.

Below we consider two cases.

$\mathrm{C}$ as e (I): $w_{1}, w_{2} \neq 0$. We shall see that in this case we get a contradiction. In view of Lemma 4 , for $\zeta \in B$,

$$
\tanh ^{-1} r=c_{\mathcal{E}(p)}(\zeta w, w)=\varrho(h(w) \zeta, h(w)),
$$

where $h$ is the Minkowski function of $\mathcal{E}(p)$. Consequently, the points $h(w) \zeta$, where $\zeta \in B$, lie on a hyperbolic circle in $E$, hyperbolically centered at $h(w)$. Therefore, this is a Euclidean circle. Moreover, $B$ is the circle given by

$$
B=\left\{\zeta=\zeta_{0}+R e^{i \theta}: 0 \leq \theta \leq 2 \pi\right\},
$$


where

Thus, for all $\zeta \in B$,

$$
\zeta_{0}=\frac{1-r^{2}}{1-r^{2} h(w)^{2}} \quad \text { and } \quad R=\frac{r}{h(w)} \cdot \frac{1-h(w)^{2}}{1-r^{2} h(w)^{2}} .
$$

$$
\zeta w_{j}=\zeta_{0} w_{j}+R_{j} e^{i\left(t+\theta_{j}\right)}, \quad t \in \mathbb{R},
$$

where $R_{j}=R\left|w_{j}\right|$ and $\theta_{j}=\arg w_{j}, j=1,2$. In view of the definition of $B$ and the equality (11) we have, for $\zeta \in B$,

$$
\begin{aligned}
\widetilde{r} & =N_{p}(\zeta w-\widetilde{w}, \widetilde{r}) \\
& =\left|\frac{\zeta_{0} w_{1}+R_{1} e^{i\left(t+\theta_{1}\right)}-\widetilde{w}_{1}}{\widetilde{r}}\right|^{2 p_{1}}+\left|\frac{\zeta_{0} w_{2}+R_{2} e^{i\left(t+\theta_{2}\right)}-\widetilde{w}_{2}}{\widetilde{r}}\right|^{2 p_{2}}, \quad t \in \mathbb{R} .
\end{aligned}
$$

By Proposition 5 we get $\widetilde{w}=\zeta_{0} w$. Therefore, in particular,

$$
\frac{1-r^{2}}{\left(1-\left|w_{2}\right|^{2 p_{2}}\right)^{1 / p_{1}}-r^{2}\left|w_{1}\right|^{2}}\left(1-\left|w_{2}\right|^{2 p_{2}}\right)^{1 / p_{1}} w_{1}=\frac{1-r^{2}}{1-r^{2} h(w)^{2}} w_{1} .
$$

From the last equality we get (remember that $0<r<1, w_{1} \neq 0$ )

$$
\left(1-\left|w_{2}\right|^{2 p_{2}}\right)^{1 /\left(2 p_{1}\right)} h(w)=\left|w_{1}\right| .
$$

Equivalently

$$
h\left(\frac{\left(1-\left|w_{2}\right|^{2 p_{2}}\right)^{1 /\left(2 p_{1}\right)}}{\left|w_{1}\right|} w\right)=1,
$$

so that (remember that $h$ is the Minkowski function of $\mathcal{E}(p)$ )

$$
1-\left|w_{2}\right|^{2 p_{2}}+\frac{\left(1-\left|w_{2}\right|^{2 p_{2}}\right)^{2 p_{2} /\left(2 p_{1}\right)}}{\left|w_{1}\right|^{2 p_{2}}}\left|w_{2}\right|^{2 p_{2}}=1
$$

and finally (remember that $w_{2} \neq 0$ !) $1=\left|w_{1}\right|^{2 p_{1}}+\left|w_{2}\right|^{2 p_{2}}$, so $w \in \partial \mathcal{E}(p)$-a contradiction.

Case (II): $w_{1}=0, w_{2} \neq 0$. We know that (see $\left.(12)\right) B_{\mathcal{E}_{\mathcal{E}(p)}^{*}}\left(\left(0, w_{2}\right), r\right)=$ $\mathcal{E}_{p}\left(\left(0, \widetilde{w}_{2}\right), \widetilde{r}, \widetilde{r}\right)$, where

$$
\widetilde{w}_{2}=\frac{w_{2}\left(1-r^{2}\right)}{1-r^{2}\left|w_{2}\right|^{2}} .
$$

Consider the geodesics (see Theorem 2)

$\varphi_{\alpha_{2}}(\lambda)$

$$
:=\left(\frac{\left(\left(1-\left|w_{2}\right|^{2 p_{2}}\right)\left(1-\alpha_{2}^{2}\left|w_{2}\right|^{2 p_{2}}\right)\right)^{1 /\left(2 p_{1}\right)}}{\left(1-\left|w_{2}\right|^{2 p_{2}} \alpha_{2} \lambda\right)^{1 / p_{1}}} \lambda, w_{2}\left(\frac{1-\alpha_{2} \lambda}{1-\left|w_{2}\right|^{2 p_{2}} \alpha_{2} \lambda}\right)^{1 / p_{2}}\right)
$$

for $\alpha_{2} \in[-1,1]$. We see that $\varphi_{\alpha_{2}}(0)=\left(0, w_{2}\right)$. Therefore the points $\varphi_{\alpha_{2}}(r)$ for all $\alpha_{2} \in[-1,1]$ are in $\partial B_{c_{\mathcal{E}(p)}^{*}}\left(\left(0, w_{2}\right), r\right)=\partial \mathcal{E}_{p}\left(\left(0, \widetilde{w}_{2}\right), \widetilde{r}, \widetilde{\widetilde{r}}\right)$. Then for 
$\alpha_{2} \in[-1,1]$ we get

$$
\begin{aligned}
\widetilde{r}= & N_{p}\left(\varphi_{\alpha_{2}}(r)-\left(0, \widetilde{w}_{2}\right), \widetilde{r}\right) \\
= & \frac{\left(1-\left|w_{2}\right|^{2 p_{2}}\right)\left(1-\alpha_{2}^{2}\left|w_{2}\right|^{2 p_{2}}\right) r^{2 p_{1}}}{\left(1-\left|w_{2}\right|^{2 p_{2}} \alpha_{2} r\right)^{2} \widetilde{r}^{2 p_{1}}} \\
& +\frac{\left|w_{2}\right|^{2 p_{2}}}{\widetilde{r}^{2 p_{2}}}\left|\left(\frac{1-\alpha_{2} r}{1-\left|w_{2}\right|^{2 p_{2}} \alpha_{2} r}\right)^{1 / p_{2}}-\frac{1-r^{2}}{1-r^{2}\left|w_{2}\right|^{2}}\right|^{2 p_{2}} .
\end{aligned}
$$

One checks that the first summand on the right hand side of (13) increases in $\alpha_{2}$ for $\alpha_{2}<r$ and decreases for $\alpha_{2}>r$. Since the expression in the second summand with exponent $1 / p_{2}$ decreases in $\alpha_{2}$ and the sum in (13) is constant we see that the second summand must be zero for $\alpha_{2}=r$.

Let us differentiate (13) with respect to $\alpha_{2}$ where it is possible (the only exception points are $\alpha_{2}=r$ if $\left.p_{2}=1 / 2\right)$. Then we get

$$
\begin{aligned}
\frac{\left(1-\left|w_{2}\right|^{2 p_{2}}\right) r^{2 p_{1}}}{\widetilde{r}^{2 p_{1}}} & \frac{2\left|w_{2}\right|^{2 p_{2}}\left(r-\alpha_{2}\right)}{\left(1-\left|w_{2}\right|^{2 p_{2}} \alpha_{2} r\right)^{3}} \\
\pm \frac{\left|w_{2}\right|^{2 p_{2}}}{\widetilde{r}^{2 p_{2}}} 2 p_{2}\left|\left(\frac{1-\alpha_{2} r}{1-\left|w_{2}\right|^{2 p_{2}} \alpha_{2} r}\right)^{1 / p_{2}}-\frac{1-r^{2}}{1-r^{2}\left|w_{2}\right|^{2}}\right|^{2 p_{2}-1} & \times \frac{1}{p_{2}}\left(\frac{1-\alpha_{2} r}{1-\left|w_{2}\right|^{2 p_{2}} \alpha_{2} r}\right)^{1 / p_{2}-1} \frac{r\left(\left|w_{2}\right|^{2 p_{2}}-1\right)}{\left(1-\left|w_{2}\right|^{2 p_{2}} \alpha_{2} r\right)^{2}} \equiv 0
\end{aligned}
$$

for all possible $\alpha_{2}$, the sign being + for $\alpha_{2}<r$ and - for $\alpha_{2}>r$.

Since all the functions appearing in (14) are real analytic for $1 / r>$ $\alpha_{2}>r$, the limit of (14) as $\alpha_{2} \rightarrow 1 / r$ must be zero, which however may hold only for $p_{2}=1$. This gives us the first part of the theorem for $n=2$.

To get the second part of the theorem we proceed as follows. Keeping in mind that $n=2, p_{2}=1, p_{1} \geq 1 / 2$ we take a point $w=\left(0, w_{2}\right)$ and find conditions equivalent to the points $\left(z_{1}, z_{2}\right) \in \mathcal{E}(p)$ lying in $\partial B_{c_{\mathcal{E}(p)}^{*}}(w, r)$. We have (for the description of automorphisms of ellipsoids in $\mathbb{C}^{2}$ with $p_{2}=1$, which we use below, see e.g. [JP])

$$
\begin{aligned}
r & =c_{B_{\mathcal{E}(p)}}^{*}\left(\left(0, w_{2}\right),\left(z_{1}, z_{2}\right)\right) \\
& =c_{B_{\mathcal{E}(p)}}^{*}\left((0,0),\left(\left(\frac{1-\left|w_{2}\right|^{2}}{\left(1-\bar{w}_{2} z_{2}\right)^{2}}\right)^{1 /\left(2 p_{1}\right)} z_{1}, \frac{z_{2}-w_{2}}{1-\bar{w}_{2} z_{2}}\right)\right),
\end{aligned}
$$

which is equivalent to (for a convex ellipsoid the function $c_{\mathcal{E}(p)}^{*}(0, \cdot)$ of any point is equal to the value of the Minkowski function at the point)

$$
\frac{\left(1-\left|w_{2}\right|^{2}\right)\left|z_{1}\right|^{2 p_{1}}}{\left|1-\bar{w}_{2} z_{2}\right|^{2} r^{2 p_{1}}}+\frac{\left|z_{2}-w_{2}\right|^{2}}{\left|1-\bar{w}_{2} z_{2}\right|^{2} r^{2}}=1 .
$$


Consequently, after some calculations,

$$
\frac{\left(1-r^{2}\left|w_{2}\right|^{2}\right)\left|z_{1}\right|^{2 p_{1}}}{r^{2 p_{1}}\left(1-\left|w_{2}\right|^{2}\right)}+\frac{\left|z_{2}-w_{2} \frac{1-r^{2}}{1-r^{2}\left|w_{2}\right|^{2}}\right|^{2}\left(1-r^{2}\left|w_{2}\right|^{2}\right)^{2}}{r^{2}\left(1-\left|w_{2}\right|^{2}\right)^{2}}=1 .
$$

So a necessary and sufficient condition for the points $\left(z_{1}, z_{2}\right) \in \partial B_{c_{\mathcal{E}(p)}^{*}}(w, r)$ to be in $\partial \mathcal{E}_{p}(\widetilde{w}, \widetilde{r}, \widetilde{r})(\widetilde{w}$ is given before) for some $\widetilde{r}, \widetilde{r}>0$ is that

$$
\widetilde{\widetilde{r}} \widetilde{r}^{2 p_{1}}=\frac{r^{2 p_{1}}\left(1-\left|w_{2}\right|^{2}\right)}{1-r^{2}\left|w_{2}\right|^{2}}, \quad \widetilde{\widetilde{r}} \widetilde{r}^{2}=\frac{r^{2}\left(1-\left|w_{2}\right|^{2}\right)^{2}}{\left(1-r^{2}\left|w_{2}\right|^{2}\right)^{2}},
$$

which gives us the second part of the theorem (after application of Lemma 6 ).

Now we start the proof of the general case (i.e. $n \geq 3, p_{j} \neq 1$ for $j=1, \ldots, n)$. Suppose that the theorem does not hold, so there is some $w \neq 0$ satisfying (1). Without loss of generality we may assume that

$$
w_{1} \neq 0 \text {. }
$$

Define

$$
\begin{aligned}
\Lambda: \mathbb{C}^{2} & \ni z \\
& \rightarrow\left(\frac{\mathbb{C}^{n} \ni z \rightarrow\left(z_{1}, z_{2}\right) \in \mathbb{C}^{2},}{\left(1-\sum_{k=3}^{n}\left|w_{k}\right|^{2 p_{k}}\right)^{1 /\left(2 p_{1}\right)}} z_{1}, \frac{1}{\left(1-\sum_{k=3}^{n}\left|w_{k}\right|^{2 p_{k}}\right)^{1 /\left(2 p_{2}\right)}} z_{2}\right) \in \mathbb{C}^{2} .
\end{aligned}
$$

We know that

$$
\begin{aligned}
L & :=(\Lambda \circ \pi)\left(B_{C_{\mathcal{E}(p)}^{*}}(w, r) \cap\left(\mathbb{C}^{2} \times\left\{\left(w_{3}, \ldots, w_{n}\right)\right\}\right)\right) \\
& =(\Lambda \circ \pi)\left(\mathcal{E}_{p}(\widetilde{w}, \widetilde{r}, \widetilde{r}) \cap\left(\mathbb{C}^{2} \times\left\{\left(w_{3}, \ldots, w_{n}\right)\right\}\right)\right)=: R .
\end{aligned}
$$

Note that

$$
\begin{aligned}
R=\Lambda\left(\left\{\left(z_{1}, z_{2}\right) \in \mathbb{C}^{2}:\left|\frac{z_{1}-\widetilde{w}_{1}}{\widetilde{r}}\right|^{2 p_{1}}+\left|\frac{z_{2}-\widetilde{w}_{2}}{\widetilde{r}}\right|^{2 p_{2}}\right.\right. & \\
& \left.\left.<\widetilde{\widetilde{r}}-\sum_{k=3}^{n}\left|\frac{w_{k}-\widetilde{w}_{k}}{\widetilde{r}}\right|^{2 p_{k}}\right\}\right)
\end{aligned}
$$

is an $N_{\left(p_{1}, p_{2}\right)}$-ellipsoid with center $\left(\widetilde{w}_{1}, \widetilde{w}_{2}\right)$ (at this point it is essential that the ellipsoids considered by us are allowed to have $\widetilde{\widetilde{r}} \neq 1$ ).

On the other hand, in view of Theorem 3,

$$
\begin{aligned}
L=\Lambda\left(\left\{\left(z_{1}, z_{2}\right):\left(z_{1}, z_{2}, w_{3}, \ldots, w_{n}\right) \in \mathcal{E}(p)\right.\right. \text { and } \\
\left.\left.\qquad c_{\mathcal{E}(p)}^{*}\left(\left(z_{1}, z_{2}, w_{3}, \ldots, w_{n}\right), w\right)<r\right\}\right)
\end{aligned}
$$




$$
\begin{aligned}
= & \Lambda\left(\left\{\left(z_{1}, z_{2}\right):\left|z_{1}\right|^{2 p_{1}}+\left|z_{2}\right|^{2 p_{2}}<1-\sum_{k=3}^{n}\left|w_{k}\right|^{2 p_{k}}\right.\right. \text { and } \\
& c_{\mathcal{E}\left(p_{1}, p_{2}\right)}^{*}\left(\left(\frac{z_{1}}{\left(1-\sum_{k=3}^{n}\left|w_{k}\right|^{2 p_{k}}\right)^{1 /\left(2 p_{1}\right)}}, \frac{z_{2}}{\left(1-\sum_{k=3}^{n}\left|w_{k}\right|^{2 p_{k}}\right)^{1 /\left(2 p_{2}\right)}}\right),\right. \\
& \left.\left.\left.\left(\frac{w_{1}}{\left(1-\sum_{k=3}^{n}\left|w_{k}\right|^{2 p_{k}}\right)^{1 /\left(2 p_{1}\right)}}, \frac{w_{2}}{\left(1-\sum_{k=3}^{n}\left|w_{k}\right|^{2 p_{k}}\right)^{1 /\left(2 p_{2}\right)}}\right)\right)<r\right\}\right) \\
= & B_{c_{\mathcal{E}\left(p_{1}, p_{2}\right)}^{*}}\left(\left(\frac{w_{1}}{\left(1-\sum_{k=3}^{n}\left|w_{k}\right|^{2 p_{k}}\right)^{1 /\left(2 p_{1}\right)}}, \frac{w_{2}}{\left(1-\sum_{k=3}^{n}\left|w_{k}\right|^{2 p_{k}}\right)^{1 /\left(2 p_{2}\right)}}\right), r\right) .
\end{aligned}
$$

And now $L=R$ is an $N_{\left(p_{1}, p_{2}\right)}$-ellipsoid, which in view of the theorem in case $n=2$ contradicts (15).

Acknowledgments. The author would like to express his gratitude to Professors Binyamin Schwarz and Uri Srebro as well as to Professor Peter Pflug for their stimulation to writing this paper and their helpful remarks.

\section{References}

[JP] M. Jarnicki and P. Pflug, Invariant Distances and Metrics in Complex Analysis, de Gruyter, 1993.

[JPZ] M. Jarnicki, P. Pflug and R. Zeinstra, Geodesics for convex complex ellipso$i d s$, Ann. Scuola Norm. Sup. Pisa 20 (1993), 535-543.

[L] L. Lempert, La métrique de Kobayashi et la représentation des domaines sur la boule, Bull. Soc. Math. France 109 (1981), 427-479.

[R] W. Rudin, Function Theory in the Unit Ball of $\mathbb{C}^{n}$, Grundlehren Math. Wiss. 241, Springer, 1980.

[Sc] B. Schwarz, Carathéodory balls and norm balls of the domain $H=\left\{\left(z_{1}, z_{2}\right) \in\right.$ $\left.\mathbb{C}^{2}:\left|z_{1}\right|+\left|z_{2}\right|<1\right\}$, Israel J. Math. 84 (1993), 119-128.

[Sc-Sr] B. Schwarz and U. Srebro, Carathéodory balls and norm balls in $H_{p, n}=$ $\left\{z \in \mathbb{C}^{n}:\|z\|_{p}<1\right\}$, preprint.

[Sr] U. Srebro, Carathéodory balls and norm balls in $H=\left\{z \in \mathbb{C}^{n}:\|z\|_{1}<1\right\}$, Israel J. Math. 89 (1995), 61-70.

[Z] W. Zwonek, Carathéodory balls and norm balls of the domains $H_{n}=\left\{z \in \mathbb{C}^{n}\right.$ : $\left.\left|z_{1}\right|+\ldots+\left|z_{n}\right|<1\right\}$, ibid. 89 (1995), 71-76.

Institute of Mathematics

Jagiellonian University

Reymonta 4

30-059 Kraków, Poland

E-mail: zwonek@im.uj.edu.pl 